\title{
Synchronism in a Metapopulation Model
}

\author{
Jacques Aveline Loureiro da Silva Manuela Longoni de Castro \\ Curso de Pós Graduação em Matemática Aplicada \\ Universidade Federal do Rio Grande do Sul
}

During the past few years there has been a growing interest in studies of population dynamics in spatial models. In a recent paper, Solè and Gamarra (1998) obtained a very simple condition for the stability of the synchronized state (all local populations oscillate in synchrony) involving just two key parameters: the fraction of individuals that disperse to nearby patches per generation and the local exponential rate of separation between close orbits (Lyapunov expoent) of the uncoupled population dynamics. The main restriction to the above result is that it was established only for an ensemble of two local populations. The metapopulation size (number of subpopulations) can play a decisive role in the ensemble persistence. In fact, Commins et al. (1992) have shown by means of numerical simulations in a host-parasitoid metapopulation model that the probability of extinction of the ensemble decreases with the metapopulation size Of course, this result is of great importance to conservation of species issues, since it relates extinction likelyhood with the number of habitat fragments forming the whole population

Given the importance of these results we propose in this paper to extend Sole and Gamarra's result to a more general metapopulation We consider a spatially explicit metapopulation model with interaction among the two nearest neighbors to relate, with a simple mathematical expression, chaos in the local, uncoupled populations, the degree of interaction among patches, size of the metapopulation and the stability of the synchronized atractor. We have obtained a necessary condition for the stability of the synchronized state involving the same key parameters of Sole and Gamarra's introducing only the size of metapopulation $n$. Since synchronism is strongly correlated with extinction, our results can provide useful information on factors leading to population extinction

\section{References}

Commins, H N., M P Hassel, and R M May (1992) The spatial dynamics of host parasitoid systems J. Anim. Ecol. 61,735-748

Solé, R V and J P G Gamarra (1998) Chaos, dispersal and extinction in coupled ecosystems $f$. Theor. Biol. 193, 539-541 